\title{
Personas atendidas por tuberculosis ocular en Colombia
}

\section{People treated for ocular tuberculosis in Colombia}

\author{
Angie T. Perilla-Rodríguez, Ángela M. Morales-Méndez, Kelly J. Ortega-Figueroa, Paula X. Vela-Peña, \\ Edgar Ibáñez-Pinilla y Diana García-Lozada* \\ Facultad de Medicina, Universidad El Bosque, Bogotá, Colombia
}

\begin{abstract}
Resumen
Introducción: La tuberculosis ocular (TBO) puede afectar cualquier parte del ojo, con o sin compromiso sistémico. A pesar de la gran cantidad de investigaciones relacionadas con la carga de la tuberculosis (TB) en el mundo, como causa principal de morbilidad y mortalidad, son escasos los estudios epidemiológicos sobre la infección ocular. Objetivo: Determinar la frecuencia de atenciones por TBO en población general y en pacientes con TB, según el Registro Individual de Prestación de Servicios de Salud (RIPS) de Colombia, entre 2013 y 2017. Método: Estudio observacional y retrospectivo, basado en fuentes secundarias de información. Se obtuvieron datos de atenciones por TBO según el RIPS, código diagnóstico A18.5. Se utilizaron como denominadores las proyecciones del Departamento Administrativo Nacional de Estadística y datos de TB del SIVIGILA. Resultados: Se identificaron 137 atenciones por TBO entre 2013 y 2017. La tasa global fue de 2.8 personas atendidas por cada millón de habitantes. Hubo relación inversa con el año evaluado $(p=0.008)$. En las mujeres fue 3.1 y en los hombres 2.5 por millón de habitantes $(p=0.275)$. Las mayores tasas se encontraron en el grupo de 75-79 años (10.2 por cada millón) y en los departamentos de Putumayo y Santander (5.8 por millón de habitantes cada uno). En pacientes con TB, la frecuencia de TBO fue de 0.2 por cada cien. Conclusiones: La frecuencia en Colombia de atenciones por TBO en pacientes con TB fue inferior a las reportadas en otros países, probablemente por un subregistro del número de personas atendidas.
\end{abstract}

Palabras clave: Tuberculosis ocular. Colombia. Bases de datos factuales. Epidemiología. Servicios de salud.

\begin{abstract}
Introduction: Ocular tuberculosis (OTB) can affect any part of the eye, with or without systemic compromise. Despite the large number of investigations related to the burden of tuberculosis (TB) in the world, as a leading cause of morbidity and mortality, few epidemiologic studies on ocular infection have been made. Objective: To determine the frequency of OTB consultations in the general population and in patients with TB, according to the personal health records system (Registro Individual de Prestaciones de Servicios de Salud [RIPS]) between 2013 and 2017 in Colombia. Method: Observational and retrospective study, based on secondary sources of information. Data of patients diagnosed with OTB were obtained from the RIPS, diagnostic code A18.5. The National Statistics Department (Departamento Administrativo Nacional de Estadística) projections and SIVIGILA data, were used as denominators. Results: There were 137 people with OTB between 2013 and 2017. The global rate was 2.8 people attended per million inhabitants; there was an inverse relationship with the year evaluated $(p=0.008)$. In women it was 3.1 and in men 2.5 per million inhabitants $(p=0.275)$. The highest rates were observed in the 75-79 age group (10.2 per million) and in the departments of Putumayo and Santander (5.8 per million inhabitants each).
\end{abstract}

Correspondencia:

*Diana García-Lozada

Avda. Cra 9, 131 A-02, Edificio Fundadores, 5. piso Fecha de recepción: 22-09-2020

110121, Bogotá D.C., Colombia

Fecha de aceptación: 16-03-2021

Disponible en internet: 01-07-2021

E-mail: dgarcialo@unbosque.edu.co

DOI: 10.24875/RMO.M21000172

Rev Mex Oftalmol. 2021;95(4):149-156

www.rmo.com.mx

0187-4519/@ 2021 Sociedad Mexicana de Oftalmología. Publicado por Permanyer. Este es un artículo open access bajo la licencia CC BY-NC-ND (http://creativecommons.org/licenses/by-nc-nd/4.0/). 
In patients with TB, the frequency of the ocular form was 0.2 per hundred. Conclusions: The frequency in Colombia of people attended for OTB in TB patients was lower than that reported worldwide, perhaps due to underreporting of the number of people attended.

Key words: Ocular tuberculosis. Colombia. Factual databases. Epidemiology. Health services.

\section{Introducción}

El microorganismo Mycobacterium tuberculosis es el agente patógeno que ocasiona la tuberculosis (TB), enfermedad infectocontagiosa bacteriana que se transmite a través de las vías respiratorias por inhalación de aerosoles o fluidos de una persona que tenga TB activa ${ }^{1}$. El principal órgano afectado es el pulmón ${ }^{2} \mathrm{y}$, en localizaciones extrapulmonares, se pueden ver comprometidos la pleura, los ganglios linfáticos, el sistema genitourinario, los huesos, las meninges y la piel, entre otros $^{3}$. Los factores que predisponen a un mayor riesgo de padecer esta enfermedad son las condiciones socioeconómicas bajas, la inmunosupresión y la etnia ${ }^{1,2}$. En todo el mundo, dos billones de personas están infectadas con $M$. tuberculosis, cada año 50 millones de pacientes sufren TB activa y alrededor de un millón de personas mueren como consecuencia de la enfermedad $^{4}$. Para el año 2018 enfermaron 10 millones de personas y la mayor parte de los casos estimados correspondieron a las regiones de Asia Sudoriental (44\%), África (24\%) y Pacífico Occidental (18\%), y en menor proporción a las regiones del Mediterráneo Oriental $(8 \%)$, Europa (3\%) y las Américas $(3 \%)^{5}$.

En 2018, en Colombia, se notificaron al Sistema Nacional de Vigilancia en Salud Pública (SIVIGILA) 14,446 casos de TB todas las formas, con una incidencia para el país de 26.9 casos por 100,000 habitantes; el $66 \%$ de los casos fueron de sexo masculino, el $21.3 \%$ eran personas de 25 a 34 años y el $53 \%$ pertenecían al régimen subsidiado ${ }^{6}$.

Según la American Academy of Opthalmology, la TB ocular (TBO) puede afectar cualquier parte del ojo (intraocular, superficial o circundante), con o sin compromiso sistémico, ya que hasta el $60 \%$ de los pacientes con evidencia de TB extrapulmonar pueden no haber sido diagnosticados de TB pulmonar. La afectación ocular puede ocurrir como una infección primaria en la conjuntiva, la córnea y la esclerótica, o como una infección secundaria desde un sitio distante, a través de la diseminación hematógena, que afecta la úvea y la retina ${ }^{7}$; la uveítis puede ser la primera manifestación de una infección oculta ${ }^{8}$.

Los datos epidemiológicos sobre TBO son escasos y poco confiables, debido a la falta de criterios diagnósticos estandarizados y a la dificultad para confirmarla por métodos de laboratorio ${ }^{9}$. El grupo del Collaborative Ocular Tuberculosis Study (COTS) menciona que el método de referencia es la detección de M. tuberculosis en tejidos o líquidos oculares, lo que rara vez se logra debido a la baja carga tisular del bacilo y al pequeño tamaño de las muestras que se obtienen de tejido ocular. Por lo tanto, el diagnóstico suele ser presuntivo, basado en los factores epidemiológicos locales, el fenotipo ocular y el resultado de pruebas inmunológicas que lo corroboren (prueba cutánea de tuberculina, ensayo de liberación de interferón-gamma [IGRA] o ambos) y de la radiografía de tórax ${ }^{10}$.

La búsqueda realizada por los autores no halló datos de prevalencias poblacionales de TBO. Respecto a la frecuencia de TBO en los pacientes con TB, se han publicado pocas investigaciones y con resultados variables, que han ido desde el $18.8 \%$ en Colombia ${ }^{11}$ y el $18 \%$ en España ${ }^{12}$ hasta el $1.39 \%$ en India ${ }^{13}$. El estudio más grande fue el de Donahue ${ }^{14}$ en 1967, en los Estados Unidos de Norteamérica, que reportó una prevalencia del $1.46 \%$ en 10,524 pacientes con TB. La publicación más reciente es un estudio en un hospital de Filipinas ${ }^{15}$, que halló una prevalencia del $6.8 \%$ (intervalo de confianza del 95\%: 2.8-13.5).

El Registro Individual de Prestación de Servicios de Salud (RIPS) es una base de datos que hace parte del Sistema Integral de Información de la Protección Social (SISPRO) del Ministerio de Salud de Colombia. En él se registran los servicios prestados en el país en cada año, ya sean procedimientos, consulta externa, hospitalización o urgencias. Es una herramienta de recolección de información de obligatorio cumplimiento, reglamentado mediante la Resolución 3374 de $2000^{16}$, y puede ser una valiosa estrategia de vigilancia de eventos no incluidos en las fichas de notificación del SIVIGILA, como es el caso de la TBO. Aunque existen deficiencias en la calidad de los datos obtenidos por el RIPS, más del $83 \%$ de los registros son útiles para las actividades de vigilancia en salud pública ${ }^{17}$.

El presente estudio buscó determinar la frecuencia de personas atendidas con diagnóstico de TBO en Colombia según el RIPS, entre los años 2013 y 2017, 
tanto en población general como en pacientes con TB, teniendo en cuenta variables sociodemográficas tales como departamento de residencia, sexo, edad y afiliación a regímenes de salud.

\section{Método}

Se realizó un estudio observacional y retrospectivo, con base en fuentes secundarias, mediante consulta en el RIPS del número de personas atendidas con diagnóstico de TBO. La búsqueda se realizó el 26 de octubre de 2018 con el código A18.5 de la Clasificación Internacional de Enfermedades (CIE-10) ${ }^{18}$ en el diagnóstico principal (afección principal diagnosticada al final del proceso de atención de la salud o de la consulta, como la causante primaria de la solicitud de atención del paciente), con fecha de atención entre los años 2013 y 2017.

Los datos de los usuarios atendidos que se incluyeron en el estudio fueron los siguientes: edad (en valores enteros en el momento de la atención, se presenta agrupada en quinquenios), sexo (según la condición física de hombre o mujer), departamento (el de residencia habitual, el lugar donde la persona lleva 6 meses o más viviendo de manera permanente; en caso de extranjeros no identificados, se diligencia el código del municipio donde se realiza la atención) y tipo de usuario (según el tipo de afiliación al Sistema General de Seguridad Social en Salud: régimen contributivo, conocido como aquella vinculación de los individuos y sus familias a través de un aporte económico financiado por el afiliado o en concurrencia entre este y su empleador, o régimen subsidiado, que acoge a aquellas personas que no pueden pagar su afiliación y esta se mantiene gracias al subsidio del Estado) ${ }^{19}$.

Para determinar el número de personas atendidas con relación a la población colombiana se utilizaron como denominadores las proyecciones de población nacional y departamental del Departamento Administrativo Nacional de Estadística (DANE). El número de personas atendidas por TBO en el periodo de 5 años se dividió entre el promedio de la población estimada para el quinquenio evaluado.

También se calculó la frecuencia de atenciones por TBO en pacientes diagnosticados con TB, utilizando como denominador los diagnósticos principales relacionados con TB en el RIPS. Los códigos CIE-10 relacionados con TB que fueron incluidos para la búsqueda en el RIPS fueron los siguientes: A150, A151, A152, A153, A154, A155, A156, A157, A158, A159, A160, A161, A162, A163, A164, A165, A167, A168, A169, A170 A171,
A178, A179, A180, A181, A182, A183, A184, A185, A186, A187, A188, A189, A190, A191, A192, A198, A199, JG5X, K230, K673, K930, M011, M490, M900, N333, N740, N741, 0980 y $\mathrm{P} 370$.

Para el cálculo de las atenciones específicas por departamento, sexo y tipo de aseguramiento se excluyeron los datos no definidos, no reportados o registrados como «otro".

La información se analizó con el programa Microsoft Excel 2018. La comparación de proporciones de TB y TBO por sexo y tipo de régimen de salud se hizo con la prueba binomial en Epidat 3.1; se utilizó el coeficiente de Spearman para la correlación entre la edad y la TBO. El nivel de significancia utilizado fue del $5 \%$. El mapa de distribución de TBO en los departamentos de Colombia se elaboró con el programa ArcMap 10.5.1, de acuerdo con la información del Geoportal DANE - Marco Geoestadístico Nacional (MGN) (https:// geoportal.dane.gov.co/v3).

\section{Resultados \\ TBO en Colombia}

La búsqueda del número de personas atendidas por TBO dio como resultado 137 (el 55.5\% mujeres). La frecuencia estimada para el periodo 2013-2017 fue de 2.8 por millón de habitantes, 3.1 en mujeres y 2.5 en hombres $(p=0.275)$. El total por cada sexo puede no corresponder a la sumatoria ya que una persona que haya sido atendida en más de un año solo se cuenta una vez, o puede incluir a las personas con sexo no reportado (Tabla 1). En cuanto al comportamiento de los casos atendidos en cada año, en 2013 fue de 0.79 por cada millón de habitantes, mientras que en 2017 fue de 0.47 por millón (Fig. 1).

La tabla 2 muestra la distribución del número de atenciones por grupos de edad para los años analizados. El total en cada grupo de edad puede no corresponder a la sumatoria ya que una persona que haya sido atendida en más de un año solo se cuenta una vez.

El mayor número de personas correspondió al grupo de 75 a 79 años, con 10.2 por millón de habitantes, y el menor al grupo de 5 a 9 años, con 1.2 por millón. Hubo relación directa entre los grupos de edad y el número de atenciones (Spearman: 0.870; $p=0.000$ ).

En los departamentos de Santander y Putumayo se encontró la mayor tasa de personas atendidas, cada uno con 5.8 por millón de habitantes, y fue menor en Cauca y Norte de Santander, con 0.7 por millón de 
Tabla 1. Personas atendidas por tuberculosis ocular según sexo

\begin{tabular}{|l|l|l|l|l|l|l|l|l|}
\hline Sexo & 2013 & 2014 & 2015 & 2016 & 2017 & Total & $\begin{array}{c}\text { Personas atendidas por } \\
\text { cada millón de habitantes }\end{array}$ & $\begin{array}{c}\text { Personas atendidas por cada } \\
\text { 100 pacientes con tuberculosis }\end{array}$ \\
\hline Femenino & 17 & 22 & 16 & 14 & 16 & 76 & 3.1 & 0.2 \\
\hline Masculino & 20 & 12 & 18 & 13 & 7 & 60 & 2.5 & 0.2 \\
\hline Total & 37 & 34 & 34 & 28 & 23 & 137 & \\
\hline
\end{tabular}

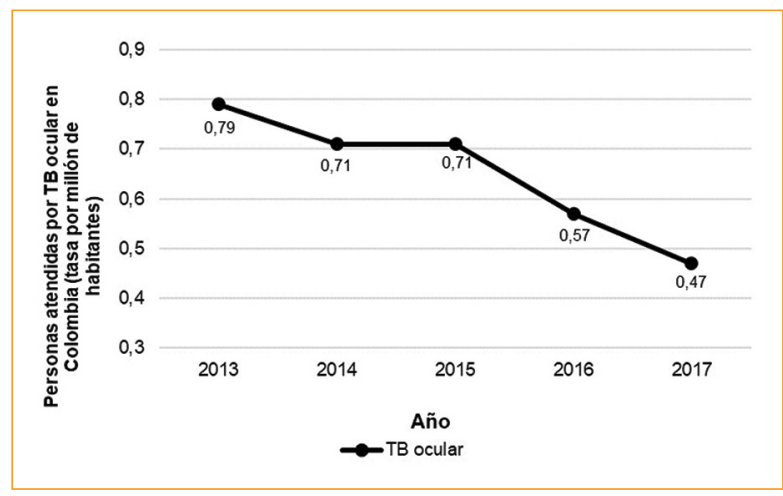

Figura 1. Personas atendidas por tuberculosis ocular (TBO) en Colombia (por millón de habitantes).

habitantes (Tabla 3). Los departamentos en color blanco en la figura 2 son los que no reportaron casos de la enfermedad.

El $63.5 \%$ de las personas con TBO estaban afiliadas al régimen contributivo. Las cifras correspondientes al promedio de afiliados a salud durante el quinquenio considerado se obtuvieron del SISPRO (consulta realizada el 5 de febrero de 2018). Hubo mayor número de diagnósticos de TBO en el régimen contributivo que en el subsidiado (4.2 vs. 2.1 por millón de habitantes, respectivamente) $(p=0.000)$.

\section{TBO en pacientes con TB}

La tasa de personas atendidas fue de 0.2 por cada cien pacientes con TB, sin diferencias por sexo $(p=0.212)$ (Tabla 1). Según el tipo de afiliación, fue de 0.3 por cada cien pacientes para el régimen contributivo y de 0.1 por cada cien pacientes para el subsidiado $(p=0.000)$. Además, se muestran los resultados por edad (Tabla 2) y departamento de residencia (Tabla 3).

Del SIVIGILA se obtuvo la información sobre los casos diagnosticados de TB extrapulmonar notificados en el periodo; el número de personas atendidas por TBO correspondió a 1.2 por cada cien diagnósticos de
TB extrapulmonar (incluyendo en el denominador los diagnósticos de meningitis tuberculosa).

\section{Discusión}

De acuerdo con los resultados de la presente investigación, la tasa de personas atendidas por TBO tuvo una tendencia a la disminución durante los años evaluados; tal comportamiento se produjo no solo por el aumento poblacional, sino también por la disminución en el número de atenciones reportadas anualmente. No obstante, no se hallaron estudios nacionales o internacionales con datos poblacionales que permitieran realizar comparaciones.

La frecuencia de TBO en los pacientes con TB fue inferior a otras reportadas previamente. Los datos del presente estudio se tomaron de los registros de las atenciones en salud que se realizan en Colombia, y los resultados de otros estudios se basaron en cifras de centros de referencia, por lo que estos últimos podrían tener un sesgo de selección ${ }^{11}$. Además de haber sido realizados en diferentes regiones y periodos, los estudios establecieron el diagnóstico de TBO según diferentes criterios clínicos, lo que podría ser otro aspecto a considerar en la variabilidad de las cifras.

Se ha mencionado que los casos de personas con TB pulmonar y extrapulmonar se han reducido con el tiempo, y que el decrecimiento en los casos extrapulmonares ha sido más lento, por lo que su proporción respecto al total de casos de TB se ha incrementado $^{3,20}$. No obstante, en este estudio no se halló un aumento en la proporción de TBO con el tiempo. Al haber encontrado frecuencias tan bajas no se puede excluir la subestimación de la etiología tuberculosa9; además, en el RIPS no se encuentra información relacionada con el tipo de pruebas de laboratorio realizadas para confirmar el diagnóstico.

De los casos notificados de TB para el año 2017 en Colombia, el $40 \%$ correspondieron a los departamentos de Antioquia (18.3\%), Valle del Cauca (13.3\%) y Bogotá $(7.8 \%)^{21}$; estas también fueron las entidades 
Tabla 2. Personas atendidas por tuberculosis ocular por grupos de edad para cada año

\begin{tabular}{|c|c|c|c|c|c|c|c|c|}
\hline Edad (años) & 2013 & 2014 & 2015 & 2016 & 2017 & Total & $\begin{array}{l}\text { Personas atendidas por } \\
\text { cada millón de habitantes }\end{array}$ & $\begin{array}{l}\text { Personas atendidas por cada } \\
100 \text { pacientes con tuberculosis }\end{array}$ \\
\hline 0 a 4 & 0 & 4 & 2 & 2 & 1 & 9 & 2.1 & 0.3 \\
\hline 5 a 9 & 1 & 1 & 2 & 2 & 0 & 5 & 1.2 & 0.3 \\
\hline 10 a 14 & 3 & 3 & 8 & 4 & 3 & 14 & 3.3 & 1.0 \\
\hline 15 a 19 & 1 & 3 & 1 & 1 & 2 & 7 & 1.6 & 0.2 \\
\hline 20 a 24 & 5 & 2 & 1 & 2 & 2 & 9 & 2.1 & 0.2 \\
\hline 25 a 29 & 2 & 3 & 2 & 1 & 0 & 7 & 1.8 & 0.1 \\
\hline 30 a 34 & 3 & 0 & 4 & 3 & 1 & 10 & 2.8 & 0.2 \\
\hline 35 a 39 & 1 & 3 & 1 & 4 & 1 & 9 & 2.8 & 0.2 \\
\hline 40 a 44 & 3 & 0 & 1 & 1 & 3 & 8 & 2.8 & 0.2 \\
\hline 45 a 49 & 5 & 1 & 2 & 1 & 0 & 9 & 3.1 & 0.2 \\
\hline 50 a 54 & 3 & 4 & 0 & 3 & 1 & 11 & 4.1 & 0.2 \\
\hline 55 a 59 & 3 & 3 & 2 & 0 & 3 & 11 & 5.0 & 0.2 \\
\hline 60 a 64 & 3 & 2 & 5 & 2 & 1 & 12 & 7.0 & 0.2 \\
\hline 65 a 69 & 1 & 0 & 0 & 1 & 3 & 5 & 3.8 & 0.1 \\
\hline 70 a 74 & 0 & 2 & 2 & 0 & 1 & 5 & 5.4 & 0.1 \\
\hline 75 a 79 & 4 & 1 & 1 & 1 & 0 & 7 & 10.2 & 0.2 \\
\hline 80 o más & 0 & 2 & 0 & 1 & 1 & 4 & 5.8 & 0.1 \\
\hline Total & 37 & 34 & 34 & 28 & 23 & 137 & & \\
\hline
\end{tabular}

territoriales en las que se encontró mayor número de personas atendidas por TBO entre 2013 y 2017. Sin embargo, la enfermedad ocular fue más frecuente en los departamentos de Santander y Putumayo; cabe mencionar que este último departamento solo reportó 553 casos de TB durante el periodo. Por otra parte, los departamentos con mayor número de atenciones por TBO en pacientes con TB fueron Bogotá y Boyacá. De los departamentos con más personas atendidas, llama la atención Bolívar, con bajas tasas de TB para el quinquenio $2010-2014^{22}$, pero fue el quinto en reportar atenciones por TBO y el tercero en personas atendidas en el país.

En seis departamentos se reportó una sola persona afectada y en 12 departamentos no se informaron casos, ocho de los cuales corresponden a departamentos en los que hubo frecuencias bajas de TB (Guainía, Vaupés, Vichada, Guaviare, Amazonas, Arauca y Casanare, además de las islas de San Andrés y Providencia). En estos departamentos, principalmente selváticos y apartados, se hubiera esperado un mayor número de atenciones de esta patología transmisible; sin embargo, los hallazgos se podrían explicar por la dificultad para el acceso a los servicios de salud o por los problemas de infraestructura que caracterizan a la región de la Amazonía-Orinoquía ${ }^{23}$, específicamente en cuanto a especialistas que pudieran detectar la forma ocular.

Con respecto al sexo, la TBO fue más frecuente en las mujeres que en los hombres, tanto en la población colombiana como en los pacientes con TB. Los hallazgos en otros estudios no han mostrado una relación entre el sexo y la $\mathrm{TBO}^{24}$, pero se podría asumir que está presente un comportamiento similar al de las TB extrapulmonares. Algunos estudios epidemiológicos con análisis multivariable han encontrado que el sexo femenino es un factor de riesgo para desarrollar TB extrapulmonar ${ }^{3,25}$.

En la población colombiana, la frecuencia de TBO se correlacionó con la edad. El grupo etario en el que se observaron más atenciones fue en el de mayores de 50 años. Se ha mencionado que la TB extrapulmonar es 
Tabla 3. Personas atendidas por tuberculosis ocular según el departamento de residencia

\begin{tabular}{|c|c|c|c|}
\hline Departamento & $\begin{array}{l}\text { Número de } \\
\text { atenciones }\end{array}$ & $\begin{array}{l}\text { Personas } \\
\text { atendidas } \\
\text { por cada } \\
\text { millón de } \\
\text { habitantes }\end{array}$ & $\begin{array}{l}\text { Personas } \\
\text { atendidas por } \\
\text { cada } 100 \\
\text { pacientes con } \\
\text { tuberculosis }\end{array}$ \\
\hline Santander & 12 & 5.8 & 0.3 \\
\hline Putumayo & 2 & 5.8 & 0.4 \\
\hline Bolívar & 10 & 4.8 & 0.4 \\
\hline Caldas & 4 & 4.1 & 0.3 \\
\hline Magdalena & 5 & 4.0 & 0.4 \\
\hline Antioquia & 24 & 3.7 & 0.1 \\
\hline Bogotá D.C. & 28 & 3.6 & 0.5 \\
\hline Valle del Cauca & 15 & 3.3 & 0.2 \\
\hline Tolima & 4 & 2.8 & 0.2 \\
\hline Boyacá & 3 & 2.4 & 0.4 \\
\hline Córdoba & 4 & 2.3 & 0.2 \\
\hline Risaralda & 2 & 2.1 & 0.1 \\
\hline Caquetá & 1 & 2.1 & 0.1 \\
\hline Cundinamarca & 5 & 1.9 & 0.2 \\
\hline Quindío & 1 & 1.8 & 0.1 \\
\hline Nariño & 3 & 1.7 & 0.1 \\
\hline Atlántico & 4 & 1.6 & 0.1 \\
\hline Sucre & 1 & 1.2 & 0.1 \\
\hline Huila & 1 & 0.9 & 0.1 \\
\hline $\begin{array}{l}\text { Norte de } \\
\text { Santander }\end{array}$ & 1 & 0.7 & 0.1 \\
\hline Cauca & 1 & 0.7 & 0.6 \\
\hline
\end{tabular}

más frecuente en los pacientes de edad avanzada debido a la desregulación del sistema inmunitario, la desnutrición y el sedentarismo, que predisponen a la infección ${ }^{26}$. Agrupando en quinquenios, el grupo de 10 a 14 años fue el de mayor número de atenciones, mucho más que en los demás grupos de niños y adolescentes. Este grupo de edad presenta riesgo para aquellos que se encuentran con desnutrición, bajo cuidado institucional 0 en situación de calle ${ }^{27}$, pero estos factores no están registrados en el RIPS de TBO. Ahora bien, entre los pacientes con TB, la frecuencia fue mayor en los menores de 14 años. Al respecto, los resultados de otros estudios han sido contradictorios. Un estudio español ${ }^{3}$ informó la asociación de la TB extrapulmonar con la edad, y un estudio realizado en Taiwán ${ }^{25}$ mostró que la edad no se asociaba con la incidencia de TB extrapulmonar; por el contrario, otro estudio en Nepal ${ }^{28}$ halló que hay mayor riesgo cuanto menor es la edad.

Por otra parte, hubo diferencia significativa según el tipo de aseguramiento, con mayor frecuencia de TBO en el régimen contributivo, a diferencia de lo que sucede con los casos de TB, que afectan en mayor proporción a los afiliados al régimen subsidiado. No se incluyeron en el estudio aspectos socioeconómicos o culturales que permitieran abordar la posible causa de estas diferencias.

Al comparar los datos de las dos principales fuentes de información de salud en Colombia, se encontró un mayor reporte de personas con TB en el RIPS $(76,812)$ que en el SIVIGILA $(60,953)$. Esto puede atribuirse a que los eventos notificados en el SIVIGILA son detectados y confirmados mediante procedimientos diagnósticos por los diferentes operadores del sistema, como parte del proceso de vigilancia; por el contrario, los datos del RIPS dependen del adecuado diligenciamiento de los profesionales que realizan las atenciones y es razonable asumir que no se hace de manera óptima, lo que puede ocasionar un sesgo de mala clasificación, además del probable subregistro, que puede ser de hasta el $30 \%$ en datos de morbilidad ${ }^{22}$. Este sesgo de información puede presentarse ya que aún existe una porción de la población colombiana sin acceso a los servicios, o también porque algunos prestadores de salud no reportan los datos de forma consistente $^{23}$. En 2013 se identificaron dificultades en la notificación y la calidad de los datos provenientes de las capitales de varios departamentos (Cauca, Caldas, Amazonas, Magdalena, Bogotá, Guaviare, Vaupés, Córdoba, Sucre, Vichada y Guainía) ${ }^{17}$; por ende, no se estaría presentando un sesgo diferencial atribuible a la calidad de los datos.

Una de las limitaciones de este estudio es que se estima un subregistro en los datos consultados, tal vez relacionado con la falta de confirmación clínica de los diagnósticos, lo que no permite apreciar la verdadera magnitud de la enfermedad. Al depender en su totalidad de fuentes de información secundarias, se puede haber presentado un sesgo de información por diligenciamiento incorrecto. Además, la información del RIPS sobre TBO no permite conocer los hallazgos oculares que fueron detectados en las personas atendidas, el ojo afectado ni las pruebas utilizadas para confirmar el diagnóstico. De hecho, el diagnóstico de TBO sigue siendo un desafío para oftalmólogos y neumólogos, 


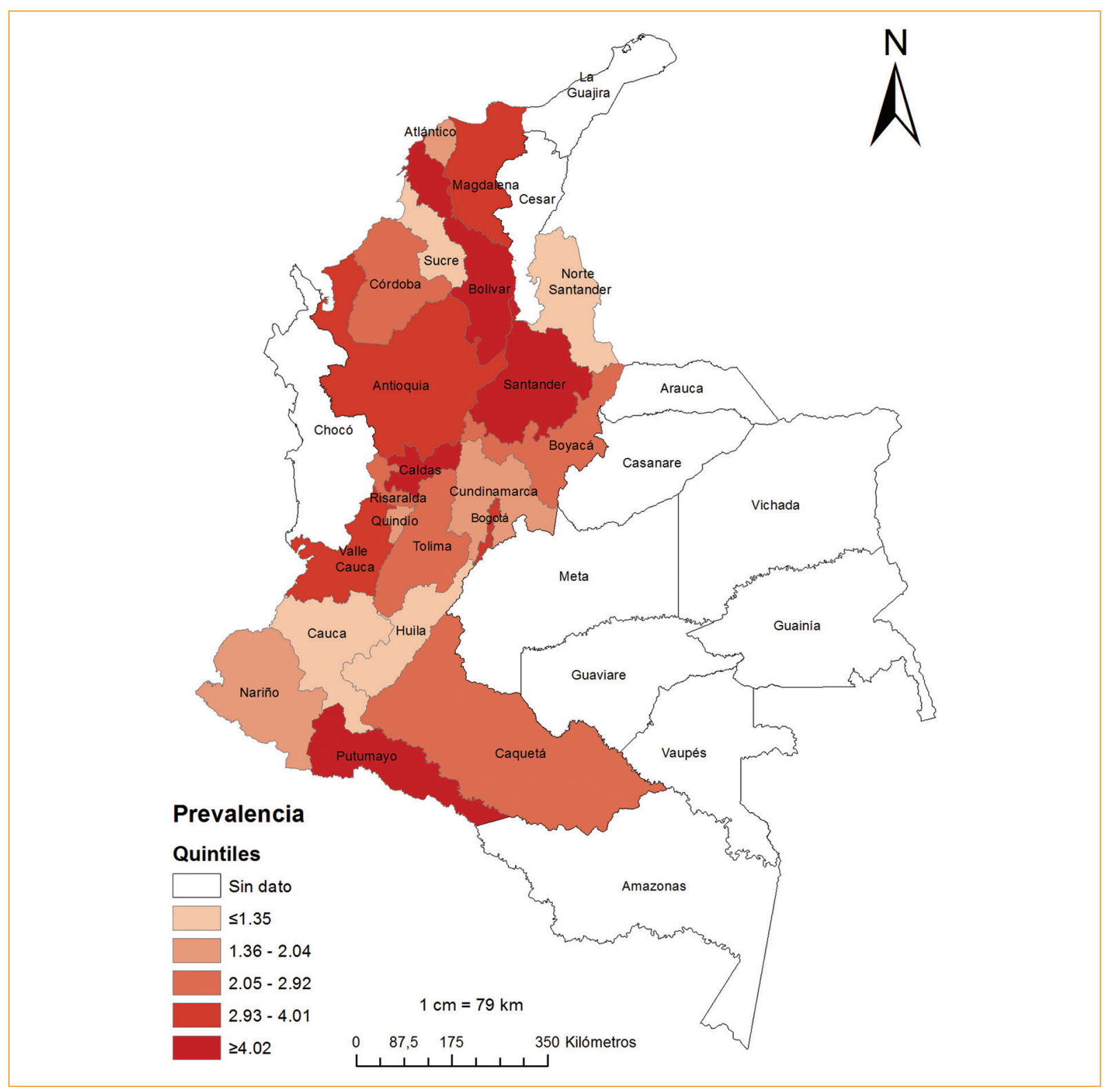

Figura 2. Personas atendidas por tuberculosis ocular en los departamentos de Colombia (tasas por millón).

debido que no se ha definido un método de referen$\mathrm{cia}^{29}$, por lo que se hace principalmente según los hallazgos clínicos. Por ello, numerosos expertos han propuesto la adopción y la divulgación de una terminología unificada que permita comprender mejor la enfermedad $^{30}$, no solo en sus aspectos clínicos, sino también epidemiológicos.

\section{Conclusiones}

Este estudio presenta las atenciones por TBO en Colombia a manera de tasas en población general y en pacientes con TB, siendo esta última considerablemente inferior a otras reportadas con anterioridad, lo que refleja un probable subregistro. Existen muy pocos datos epidemiológicos sobre la distribución de la TBO que permitan hacer comparaciones con nuestros resultados. Se debe crear conciencia entre los profesionales de la salud sobre la posibilidad de afección ocular en los pacientes con TB, para que realicen la búsqueda de signos oculares incluso en pacientes asintomáticos $y$, adicionalmente, para que efectúen el reporte en el RIPS de manera confiable. Aunque no es de las formas extrapulmonares más 
frecuentes, la TBO puede ser una manifestación primaria que permita realizar el diagnóstico sistémico.

\section{Agradecimientos}

A Laura Cabezas Pinzón, ingeniera ambiental e investigadora del Instituto de Salud y Ambiente de la Vicerrectoría de Investigaciones de la Universidad El Bosque, por su ayuda con la elaboración del mapa de distribución en los departamentos de Colombia.

\section{Financiación}

No se recibió financiación para la elaboración de esta investigación.

\section{Conflicto de intereses}

Todos los autores declaran no tener ningún tipo de conflicto de intereses.

\section{Responsabilidades éticas}

Protección de personas y animales. Los autores declaran que para esta investigación no se han realizado experimentos en seres humanos ni en animales.

Confidencialidad de los datos. Los autores declaran que en este artículo no aparecen datos de pacientes.

Derecho a la privacidad y consentimiento informado. Los autores declaran que en este artículo no aparecen datos de pacientes.

\section{Bibliografía}

1. Thomas TA. Tuberculosis in children. Thorac Surg Clin. 2019;29:109-21.

2. Shakarchi FI. Ocular tuberculosis: current perspectives. Clin Ophthalmol. 2015;9:2223-7.

3. García-Rodríguez J, Álvarez-Díaz H, Lorenzo-García M, Mariño-Callejo A, Fernández-Rial A, Sesma-Sánchez P. Extrapulmonary tuberculosis: epidemiology and risk factors. Enferm Infecc Microbiol Clin. 2011;29:502-9.

4. GBD Tuberculosis Collaborators. The global burden of tuberculosis: results from the Global Burden of Disease Study 2015. Lancet Infect Dis. 2018;18:261-84.

5. Organización Mundial de la Salud. Informe mundial sobre la tuberculosis 2019 - Sinopsis. 2019. (Consultado el 18 de octubre de 2019.) Disponible en: https://www.who.int/tb/publications/global_report/gtbr2019_ExecutiveSummary_sp.pdf?ua $=1$

6. Instituto Nacional de Salud. Colombia. López M. Informe del evento. Tuberculosis, Colombia 2018. 2019. (Consultado el 30 de septiembre de 2019.) Disponible en: https://www.ins.gov.co/buscador-eventos/Informesdeevento/TUBERCULOSIS_2018.pdf

7. American Academy of Ophhalmology. San Francisco: The Academy; 2014. Ocular tuberculosis (TB) - Asia Pacific. 2019. (Consultado el 29 de marzo de 2019.) Disponible en: https://www.aao.org/topic-detail/ocular-tuberculosis-tb--asia-pacific-2.
8. Agrawal R, Gunasekeran DV, Raje D, Agarwal A, Nguyen QD, Kon OM, et al. Global variations and challenges with tubercular uveitis in the Collaborative Ocular Tuberculosis Study. Invest Ophthalmol Vis Sci. 2018;59:4162-71.

9. Khairallah M, Jelliti B, Attia S. Uveitis in the developing world. Expert Rev Ophthalmol. 2010;5:161-76.

10. Agrawal R, Testi I, Mahajan S, Yuen YS, Agarwal A, Kon OM, et al. Collaborative Ocular Tuberculosis Study consensus guidelines on the management of tubercular uveitis - report 1: guidelines for initiating antitubercular therapy in tubercular choroiditis. Ophthalmology. 2021;128:266-76.

11. Fonseca EVF, Yunda LFI, Herrera KCM, Moreno GC. Extrapulmonary tuberculosis in Colombian children: epidemiological and clinical data in a reference hospital. Int J Mycobacteriol. 2017;6:132-7.

12. Bouza $E$, Merino $P$, Muñoz $P$, Sánchez-Carrillo $C$, Yáñez J, Cortes $C$. Ocular tuberculosis. A prospective study in a general hospital. Medicine (Baltimore). 1997;76:53-61.

13. Biswas J, Badrinath SS. Ocular morbidity in patients with active systemic tuberculosis. Int Ophthalmol. 1995;19:293-8.

14. Donahue HC. Ophthalmologic experience in a tuberculosis sanatorium. Am J Ophthalmol. 1967;64:742-8.

15. Lara LP, Ocampo V. Prevalence of presumed ocular tuberculosis among pulmonary tuberculosis patients in a tertiary hospital in the Philippines. $J$ Ophthalmic Inflamm Infect. 2013;3:1.

16. Colombia. Ministerio de Salud Pública. Resolución 3374 de 2000 (diciembre 27): Por la cual se reglamentan los datos básicos que deben reportar los prestadores de servicios de salud y las entidades administradoras de planes de beneficios sobre los servicios de salud prestados. Bogotá D.C.: 27 de diciembre de 2000. (Consultado el 26 de octubre de 2019.) Disponible en: https://www.nuevaeps.com.co/sites/default/files/inline-files/resolucion3374-de-2000.pdf

17. Martínez M, Pacheco O. Utilidad de los Registros Individuales de Prestación de Servicios (RIPS) para la vigilancia en salud pública, Colombia, 2012. Instituto Nacional de Salud; 2013. p. 176-92.

18. Organización Panamericana de la Salud. Clasificación estadística internacional de enfermedades y problemas relacionados con la salud. 10. revisión. Washington, D.C.: OPS; 2018.

19. Colombia. Ministerio de Salud y Protección Social. Oficina de Tecnología de Información y Comunicaciones. Lineamiento técnico para el registro y envío de los datos del Registro Individual de Prestaciones de Salud RIPS, desde las instituciones prestadoras de servicios de salud a las EAPB. Versión 8. 2019. (Consultado el 14 de enero de 2021.) Disponible en: https://www.minsalud.gov.co/sites/rid/Lists/BibliotecaDigital/RIDE/DE/ OT/Lineamientos-Tecnicos-para-IPS.pdf

20. Peto HM, Pratt RH, Harrington TA, LoBue PA, Armstrong LR. Epidemiology of extrapulmonary tuberculosis in the United States, 1993-2006. Clin Infect Dis. 2009;49:1350-7.

21. Instituto Nacional de Salud. Colombia. López M. Informe del evento. Tuberculosis, Colombia 2017. 2018. (Consultado el 30 de septiembre de 2019.) Disponible en: https://www.ins.gov.co/buscador-eventos/Informesdeevento/Tuberculosis\%202017.pdf

22. Pedraza A. Estimación de la carga de enfermedad por tuberculosis en Colombia, 2010 a 2014. [Tesis de maestría]. Bogotá: Facultad de Medicina, Universidad El Bosque; 2017.

23. Bernal O, Forero JC, Villamil MP, Pino R. Disponibilidad de datos y perfil de morbilidad en Colombia. Rev Panam Salud Pública. 2012;31:181-7.

24. Win M, Chee SP. Epidemiological aspects of ocular tuberculosis. En: Kumar A, Chawla R, Sharma N, editores. Ocular tuberculosis. Suiza: Springer International Publishing; 2017. p. 1-6.

25. Lin JN, Lai CH, Chen YH, Lee SS, Tsai SS, Huang CK, et al. Risk factors for extra-pulmonary tuberculosis compared to pulmonary tuberculosis. Int J Tuberc Lung Dis. 2009;13:620-5.

26. García-Goez J, Munévar H, Pacheco R. Tuberculosis en pacientes mayores de 80 años atendidos en una institución de alta complejidad. Rev Colomb Neumol. 2017;29:19-25.

27. Tovar M, Tang A, Concha N. Tuberculosis extrapulmonar en pediatría: un reto diagnóstico. Medicas UIS. 2013;2b:45-58.

28. Sreeramareddy CT, Panduru KV, Verma SC, Joshi HS, Bates MN. Comparison of pulmonary and extrapulmonary tuberculosis in Nepal - a hospital-based retrospective study. BMC Infect Dis. 2008:8:8.

29. Lee C, Agrawal R, Pavesio C. Ocular tuberculosis - a clinical conundrum. Ocul Immunol Inflamm. 2016;24:237-42.

30. Agrawal R, Agarwal A, Jabs DA, Kee A, Testi I, Mahajan S. Standardization of nomenclature for ocular tuberculosis - results of Collaborative Ocular Tuberculosis Study (COTS) Workshop. Ocul Immunol Inflamm. 2019 Dec 10;1-11. doi: 10.1080/09273948.2019.1653933. Online ahead of print. 